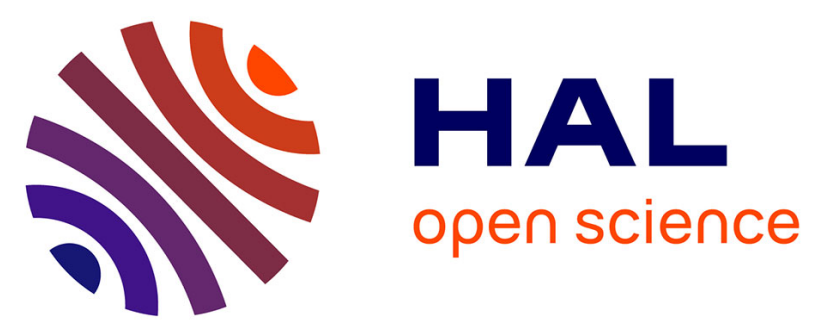

\title{
Synthesis of Functionalized Few-Layer Graphene Through Fast Electrochemical Expansion of Graphite
}

Yann Leroux, Jean-François Bergamini, Soraya Ababou-Girard, Jean-Christophe Le Breton, Philippe Hapiot

\section{- To cite this version:}

Yann Leroux, Jean-François Bergamini, Soraya Ababou-Girard, Jean-Christophe Le Breton, Philippe Hapiot. Synthesis of Functionalized Few-Layer Graphene Through Fast Electrochemical Expansion of Graphite. Journal of electroanalytical chemistry and interfacial electrochemistry, 2015, 753, pp.42-46. 10.1016/j.jelechem.2015.06.013 . hal-01163647

\section{HAL Id: hal-01163647 https://hal-univ-rennes1.archives-ouvertes.fr/hal-01163647}

Submitted on 19 Nov 2015

HAL is a multi-disciplinary open access archive for the deposit and dissemination of scientific research documents, whether they are published or not. The documents may come from teaching and research institutions in France or abroad, or from public or private research centers.
L'archive ouverte pluridisciplinaire HAL, est destinée au dépôt et à la diffusion de documents scientifiques de niveau recherche, publiés ou non, émanant des établissements d'enseignement et de recherche français ou étrangers, des laboratoires publics ou privés. 


\section{Synthesis of Functionalized Few-Layer Graphene Through Fast Electrochemical Expansion of Graphite.}

Yann R. Leroux, ${ }^{1 *}$ Jean-François Bergamini ${ }^{1}$, Soraya Ababou ${ }^{2}$, Jean-Christophe Le Breton ${ }^{2}$, Philippe Hapiot $^{1}$

${ }^{1}$ Université de Rennes 1, Sciences Chimiques de Rennes (Equipe MaCSE), CNRS, UMR 6226, Campus de Beaulieu, Bat 10C, 35042 Rennes Cedex, France

${ }^{2}$ Equipe de Physique des Surfaces et Interfaces, Institut de Physique de Rennes, UMR 6251, CNRS-

Université de Rennes 1, Campus de Beaulieu, Bât 11C, 35042 Rennes Cedex, France

*yann.leroux@univ-rennes1.fr

ABSTRACT:

Fast and reproducible production of graphene flaks from graphite is an important process considering the possible industrial applications of graphene. We propose an electrochemical method to produce functionalized few-layer graphenes in minutes, without the need of prolonged ultrasonic treatment. It mainly consists in the cathodic electrochemical expansion of graphite in DMF. Functionalization of graphite is achieved by reducing aryl diazonium salts in organic solution. This simple methodology allows easily and quickly preparation of modified few-layer graphenes. Two different graphite sources were considered in this work for electrochemical graphene flaks production.

KEYWORDS: HOPG; Graphite; Graphene; Aryldiazonium electroreduction; carbon surface modification; electrochemical exfoliation. 


\section{Introduction}

Since it's (re)discovery in 2004 [1], graphene, a two-dimensional (2D) structure consisting of $\mathrm{sp}^{2}$-bonded carbon atoms, has attracted much attention both for fundamental researches and applications because of unique structural and physical properties. [2] Since the first researches by Geim and Novoselov that have shown the peculiar properties of this new material, [1] the synthesis of graphene materials was a huge challenge. In the last 10 years, many researches aim to easily produce high quality graphene monolayer. The recent progress in graphene synthesis by Chemical Vapor Deposition (CVD) allows daily manipulations of graphene monolayer in research laboratory and means it may be used in industrial processes, which is the key step between a promising material and a new material that will change economical industries. The huge progress made by CVD technique permits the reproducible preparation of high quality graphene sheets and on a large scale. Only five years after the rush for graphene synthesis, researchers from Samsung in collaboration with other Korean laboratories were able to synthesis high quality monolayer graphene at the wafer scale,[3] as well as 30 inches flexible conductive graphene panel using a roll-to-roll process. [4] As another example of the increasing quality of graphene synthesis by CVD, Tour et al obtained a single graphene crystal with a diameter as large as $2.3 \mathrm{~mm} .[5]$

Notice that if high quality graphene is required for applications like those related to electronic devices, lower graphene quality could be appropriate in other fields (optical, mechanical, barrier...). [6] Many efforts were done in graphene synthesis via the graphene oxide route. Hummer's method [7] allows the preparation of the so-called "reduced graphene oxide": graphite is initially oxidized to obtain highly soluble graphene oxide that is reduced in a second step. This technique could produce high quantity of graphene materials and with low cost. However, the graphene flaks obtained by such method generally leads to low quality materials with many defects and vacancies because oxides always remain at the graphene surface and edges. This is why other non-oxidative techniques were proposed for preparing large quantities of graphene materials with low defects and at a low cost. Liquid exfoliation 
processes were proposed to produce pristine graphene from Highly Ordered Pyrolytic Graphite (HOPG) or graphite flakes. [8] Basically, it is to find the right conditions to weaken the strong $\pi-\pi$ inter-sheet interactions that favor staking of graphene sheets in graphite. This could be done by sonicating the graphite flakes (or HOPG) in an appropriate solvent. Efficient solvents able to disperse graphene sheets must have surface tensions equal to that of graphene in order to prevent their re-aggregation. Molecular dynamics simulations [9] revealed that the prevailing barrier hindering the aggregation of graphene is the last layer of solvent molecules confined between the graphene sheets, which results from the strong affinity of the solvent molecules for graphene. Prediction of the potential solvents able to disperse graphene is consistent with the widespread use of NMP, DMF and DMSO for this purpose. To prevent the re-aggregation of graphene sheets in solution, surfactants could also be added [10] with the limitation that the removal of the surfactants is often a tedious task. Also despite its simplicity, the major drawback of this technique is that prolonged ultrasonic treatment is necessary, which could damage the obtained graphene flaks and requires several hours of treatment ( $\geq 10$ hours) to be efficient.

Electrochemical synthesis of graphene was proposed as an alternative method to the classical liquid exfoliation. [11] Electrochemical methods could produce similar graphene quality (and quantity) of graphene in just a few minutes and fast electrochemical production of graphene flaks were recently achieved using anodic oxidation of graphite in aqueous solution [11,12] or in room temperature ionic liquids (RTILs). [13] These techniques produce important quantities of graphene in minutes, without the need of long ultrasonic treatment, but application of a high anodic potential could potentially enhance the number of defects (by incorporation of oxygen atom) in the obtained graphene flaks.

Graphite expansion (or intercalation) experiments performed in the 70's, found a second life [14] with the discovery of graphene properties. As recently shown in the literature, the utilization of graphite intercalation compounds (GIC) instead of bare graphite provides higher graphene quality. [15, 16] A convenient method is to use a cathodic electrochemical treatment coupled to a short ultrasonic step; this limits the damage and defect of the produced graphene solution. Recently, inspired by the 
electrochemical reaction of negative graphite electrodes in lithium ion batteries, utilization of graphite intercalated with lithium ions was proposed as an alternative route to produce low defect graphene solution but it still requires long ultrasonic treatment at high intensity. [14] To avoid prolonged ultrasonic treatment, Swager et al. proposed to exchange lithium ions by tetra- $n$-butyl ammonium ions which offers a simple graphene cathodic electrochemical method, even if the exchange process took 24 hours to be efficient. [17]

To increase the solubility of such liquid-exfoliated or electrochemically exfoliated graphene materials, covalent functionalization appears to be as an efficient alternative. Tour et al. [18] described a two-step protocol where thermally expanded graphite was first functionalized by bromophenyl diazonium salts and then exfoliated by sonication treatment. This method leads to more concentrated graphene solution without the need of adding any stabilizers. Hirsch et al. reported that liquid-exfoliated graphene sheets could be functionalized with 4-tert-butylphenyl diazonium or 4-sulfonyphenyl diazonium salts, which prevents the re-aggregation of graphene materials in solution. [19]

In this study, we describe a fast electrochemical method based on the electrochemical expansion and functionalization of graphite in n,n-dimethylformamide (DMF) as useful carbon source to obtain functionalized graphene flaks in minutes with low defects.

\section{Experimental section.}

\subsection{Chemicals and reagents.}

Commercially available reagents were used as received. Tetra-n-octylammonium bromide was purchased from Alfa Aesar, 4-bromobenzenediazonium tetrafluoroborate from Fluka and n,ndimethylformamide (DMF) from Fisher chemical. $0.45 \mu \mathrm{m}$ pore size PTFE membranes were purchased from Sartorius. Flexible graphite sheets and Highly Ordered Pyrolytic Graphite (HOPG) were purchased from Goodfellow. 
2.2 Preparation of few-layer graphene.

Electrochemical expansion and functionalization was performed using a DC power supply from Schlumberger (type K18R1) in a two-electrode electrochemical system. Sonication treatment was performed using a Fisherbrand ultrasonic bath (FB15050) at a frequency of $37 \mathrm{KHz}$ and an effective power of $75 \mathrm{~W}$. All solutions were degassed with Argon prior any experiments. DMF was chosen as the electrochemical solvent due to its good solubility with respect to tetraoctylammonium bromide and graphene dispersing properties [9], as well as its large electrochemical window [20].

\subsubsection{Electrochemical expansion.}

Production of graphene flaks was performed using a two-electrode system where Highly Ordered Pyrolytic Graphite (HOPG) served as an electrode and carbon source, and a grounded platinum electrode was placed closed to the carbon source $(\geq 1 \mathrm{~cm})$. Electrochemical expansion of HOPG was performed in a DMF solution containing $0.1 \mathrm{M}$ tetra-n-octylammonium bromide as supporting electrolyte. The electrochemical expansion process was carried out by applying a DC bias of -10 V on the carbon source.

\subsubsection{Electrochemical functionalization.}

Functionalization of the graphene flaks was performed consecutively to the electrochemical expansion of HOPG. To efficiently functionalize the carbon source, the electrochemical expansion step is extended to 3 hours. Due to electrolyte decomposition at $-10 \mathrm{~V}$ the potential is reduced to $-1 \mathrm{~V}$ during electrochemical functionalization. [17]

Protocol 1: After expansion in DMF solution, the DC bias voltage is reduced from $-10 \mathrm{~V}$ to -1 V. $27 \mathrm{mg}$ of 4-bromobenzenediazonium tetrafluoroborate is then introduced in the DMF solution to reach a final concentration of $10^{-2} \mathrm{M}$ of aryl diazonium ion. Afterward, the DC bias voltage of $-1 \mathrm{~V}$ is 
maintained for 2 hours. Dispersion of the graphene flaks in DMF was obtained by smooth sonication for $10 \mathrm{~min}$. Filtration and purification of graphene flaks dispersion was performed using a $0.45 \mu \mathrm{m}$ pore size PTFE membrane. The obtained graphene film is then thoroughly rinsed with acetone and ethanol.

Protocol 2: After expansion in DMF solution, the carbon source is rinsed with acetone, dry with an Argon stream and then introduced in a new DMF solution containing $0.1 \mathrm{M}$ tetra- $n$-octylammonium bromide as supporting electrolyte and $10^{-2} \mathrm{M}$ of 4-bromobenzenediazonium tetrafluoroborate. A DC bias voltage of $-1 \mathrm{~V}$ is applied for 2 hours in this new solution to the carbon source. Dispersion of the graphene flaks in DMF was obtained by sonication for $10 \mathrm{~min}$. Filtration and purification of graphene flaks dispersion was performed using a $0.45 \mu \mathrm{m}$ pore size PTFE membrane. The obtained graphene film is then thoroughly rinsed with acetone and ethanol.

Dispersion of the functionalized graphene materials was performed by sonication of the obtained graphene films in ultrasonic bath during 30 min to 1 hour in DMF.

\subsection{Atomic Force microscopy (AFM).}

AFM experiments were carried out on NT-MDT Ntegra microscope in ambient conditions to obtain topographic and phase images. Semi-contact mode experiments were investigated using silicon nitride tips with a force constant of $48 \mathrm{~N} \cdot \mathrm{m}^{-1}$ and a resonance frequency around $168 \mathrm{kHz}$. Graphene solutions were drop-casted on a freshly cleaved mica surface and dry in oven at $75^{\circ} \mathrm{C}$. Samples were cleaned under a stream of Argon gas prior to imaging in order to remove dust particles. Images were analyzed with Gwyddion software. Height statistics were obtained by measuring the height of 60 different structures observed on several topographic images.

\subsection{X-ray Photoelectron Spectroscopy (XPS).}

XPS was realized using an $\mathrm{Mg} \mathrm{K} \alpha(1253.6 \mathrm{eV})$ anode source and Omicron HA100 electron energy analyzer $\left(1.0 \mathrm{eV}\right.$ resolution) at the surface normal. A pressure lower than $310^{-9}$ Torr was 
maintained during the measurements. Curve fitting of the core levels was realized with Igor software using a convolution of Gaussian and Lorentzian functions after the subtraction of an integrated background. For XPS experiments, large amount of functionalized few-layer graphene material was synthesis (100 mL solution).

\subsection{Determination of graphene concentration by UV-Visible Spectroscopy.}

Coleman and coworkers [21] show that it is possible to determine graphene solution concentration using UV-Vis spectroscopy. Optical absorbance $\left(\lambda_{\mathrm{ex}}=660 \mathrm{~nm}\right)$ divided by cell length $(\mathrm{A} / \mathrm{l})$ as a function of graphene solution concentration (C) shows Beer-Lambert behavior with an average absorption coefficient of $\left\langle\alpha_{660}\right\rangle=2460 \mathrm{~L} \mathrm{~g}^{-1} \mathrm{~m}^{-1}$, independently of the solvent used to dispersed graphene materials. In this work, we will use the same average absorption coefficient to determine graphene solution concentration, following equation (1):

$$
\mathrm{A} / \mathrm{l}=<\alpha_{660}>\mathrm{C}
$$

\section{Results and Discussion}

For practical applications, a fast cathodic electrochemical process that can be performed in minutes is necessary. As proposed in the 70's by Simonet et al., [22] fast electrochemical expansion of graphite could be performed in DMF solution containing alkykammonium ions as intercalants. As demonstrated in their work, using small tetraalkylammonium cations, as tetramethyl $\left(\mathrm{TMA}^{+}\right)$or tetraethyl $\left(\mathrm{TEA}^{+}\right)$ammonium ions, charge and discharge of graphitic materials are reversible. In the presence of bulky cations such as tetraoctylammonium $\left(\mathrm{TOA}^{+}\right)$ions, the charging/discharging is no longer reversible and a huge irreversible increase of the volume of the graphite crystal is observed. More recently, Dryfe and coworkers [23] studied the electrochemical intercalation/reduction of 
tetraalkylammonium-containing electrolytes using N-Metyl-2-Pyrrolidone (NMP) as solvent. In agreement with the work of Simonet et al., they demonstrated that charge/discharge reversibility is related to the crystallographic diameter of the tetraalkylammonium ions and that irreversible expansion of graphitic materials is obtained using large cations. In the purpose of expanding HOPG materials to obtain graphene sheets, we have considered the $\mathrm{TOA}^{+}$cation as an intercalant as the bonding between the graphite layers is irreversibly broken during the electrochemical intercalation (see Scheme 1).

Scheme 1. Scheme of the electrochemical expansion, functionalization and exfoliation of graphite into functionalized graphene flaks.

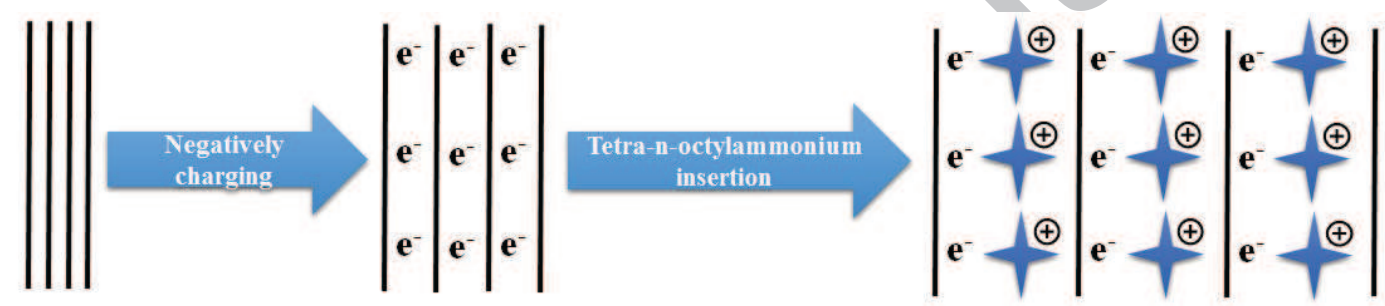

Graphite
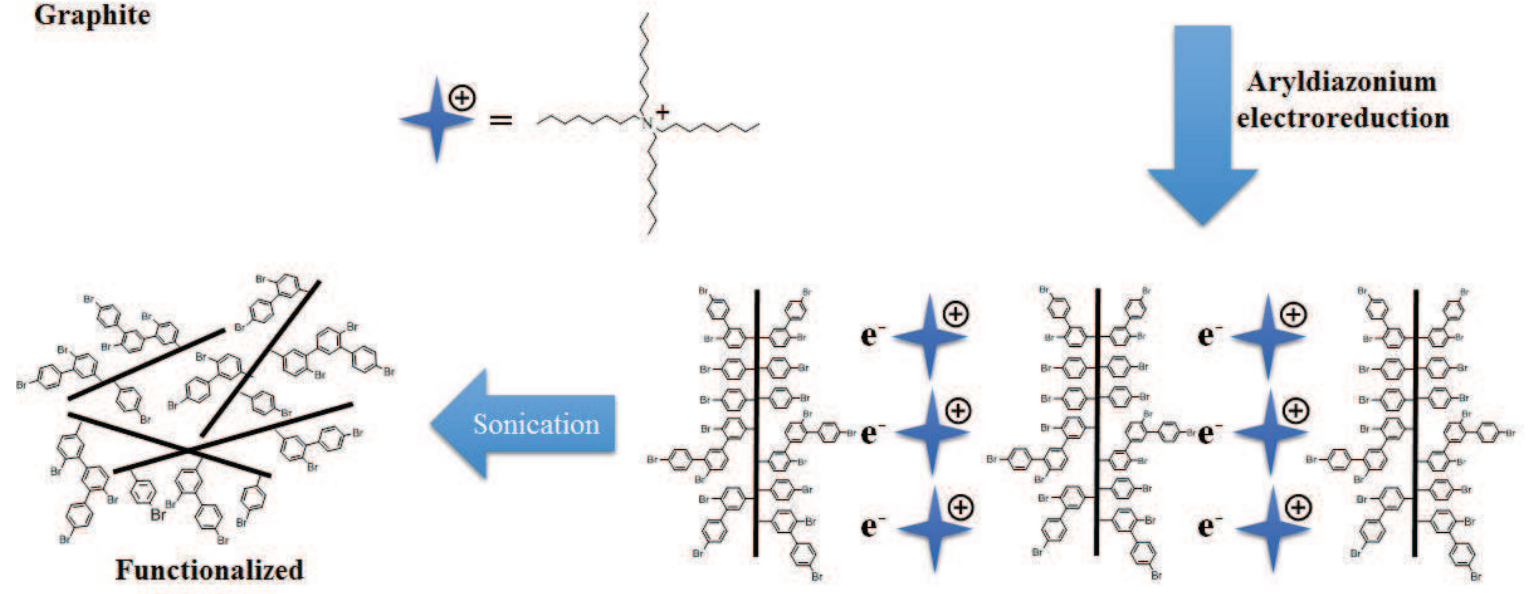

Graphene

In a first step, HOPG was immersed in a DMF solution containing $0.1 \mathrm{M}$ tetra- $n$ octylammonium bromide in a two-electrode system. A platinum wire was used as a counter electrode in this system. A DC bias voltage of $-10 \mathrm{~V}$ was applied at the HOPG electrode producing expanded HOPG. In a second step, the functionalization of the obtained expanded material was performed, 
introducing 4-bromobenzenediazonium ions $\left(\mathbf{4}-\mathbf{B r}-\mathbf{A r}-\mathbf{N}_{2}^{+}\right)$in the DMF solution. Two protocols were adopted. In the first one, HOPG was first expanded in DMF solution containing only $0.1 \mathrm{M}$ tetra- $n$ octylammonium bromide for three hours. The applied bias was then decreases from $-10 \mathrm{~V}$ to $-1 \mathrm{~V}$ and $0.01 \mathrm{M}$ of $\mathbf{4}-\mathrm{Br}-\mathrm{Ar}-\mathbf{N}_{\mathbf{2}}{ }^{+}$was introduced in the DMF solution. The DC bias was maintained at $-1 \mathrm{~V}$ for a couple of hours. The functionalized electrochemical graphene (FEG) obtained by this first protocol will be named FEG-1. In the second protocol, after expansion of HOPG in similar conditions for three hours, the expanded HOPG is rinsed with acetone and dried with an Argon stream. Then, the sample is introduced into a new freshly prepared solution of DMF containing the supporting electrolyte and the aryl diazonium ion for two hours, while applying a DC bias of $-1 \mathrm{~V}$ leading to the functionalized electrochemical graphene FEG-2. Both protocols are fully described in the experimental section part.

During the first step, graphite is negatively charged. [22] The charging of graphite implies the diffusion of the $\mathrm{TOA}^{+}$cation into the charged graphite that penetrates into the graphene lattice. At such negative potential, electro-decomposition of intercalated $\mathrm{TOA}^{+}$ions, which neutralized the accumulated negative charges into graphite lattice, occurs and thereby allows a continual maintaining of the driving force for intercalation of $\mathrm{TOA}^{+}$cations. [17] Because of the large crystallographic size $(\sim 2 \mathrm{~nm})$ of the $\mathrm{TOA}^{+}$cation compare to the interplanar spacing of graphite $(0.354 \mathrm{~nm})$ [24], intercalation of $\mathrm{TOA}^{+}$into graphite lattice leads to an irreversible breakage of the bonds between the layers. This permits an efficient graphene functionalization during aryldiazonium electro-reduction and further graphene dispersion by sonication.

X-Ray Photoelectron Spectroscopy (XPS) analysis was performed to estimate the presence of defects in the prepared graphene flaks by considering the amount of oxygen atoms in the carbon $\mathrm{sp}^{2}$ lattice and the degree of functionalization of the graphene sheets as a function of the bromine signal (see Figure 1a). As shown in Table 1, only a few percents of oxygen are present in both FEG sample (3\% maximum) after the cathodic electrochemical treatment. For comparison, Mullen et al. obtained $7.5 \%$ of oxygen using anodic electrochemical method. [12a] Moderate amount of bromine $(0.7 \%)$ is detected 
by XPS for the first modification protocol (FEG-1). The second modification protocol (FEG-2) is more efficient leading to a ratio of $1.2 \%$ of bromine. This is in accordance with others functionalized methods reported in the literature using aryl diazonium electrografting. [17, 18]

Table 1. Concentration and XPS summary of the as-prepared few-layer graphene solution.

\begin{tabular}{|c|c|c|c|c|}
\hline Sample & Concentration $^{\mathrm{a}}$ & XPS O/C ratio \% & XPS N/C ratio \% & XPS Br/C ratio \% \\
\hline FEG-1 & $8.13 \mu \mathrm{g} / \mathrm{mL}$ & 3.0 & 0.3 & 0.7 \\
FEG-2 & $17.5 \mu \mathrm{g} / \mathrm{mL}$ & 1.3 & 1.4 & 1.2 \\
\hline
\end{tabular}

${ }^{a}$ Graphene concentrations were determined by UV-Vis method developed by Hernandez et al. ${ }^{21}$

a)

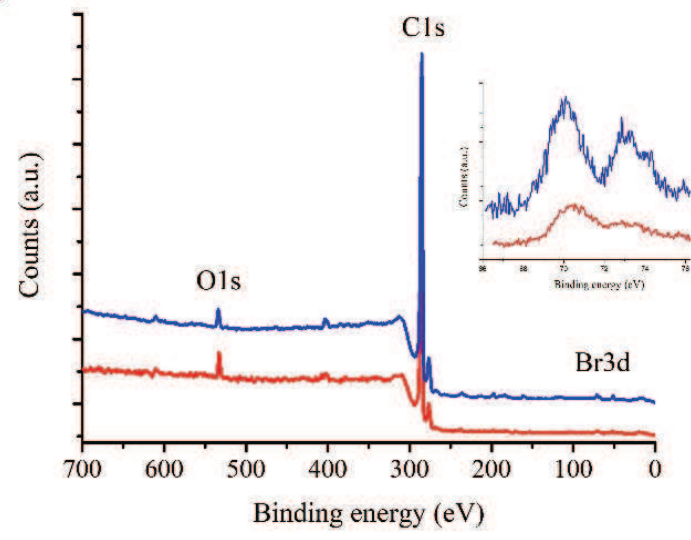

b)

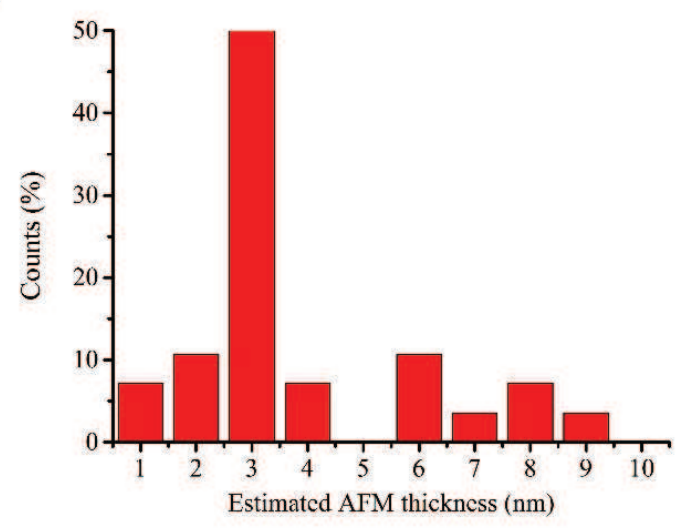

Figure 1. a) XPS scans of the FEG-1 (red line, down) and FEG-2 (blue line, top) samples. Insert: Br3d XPS spectra of FEG-1 (red line, bottom) and FEG-2 (blue line, top). b) Statistical AFM thickness analyses of FEG-2 sample.

As explained in the introduction, covalent modification of graphene materials is an efficient way for increasing graphene solubility. Concentration of FEG solutions were thus determined by UV-Vis spectroscopy [21] after three weeks of sedimentation in order to efficiently remove any graphitic particles. We observed that our FEG DMF solution could be stored at least a month without further sedimentation of the graphene material. As expected, graphene solubility is greatly enhanced compare 
too pristine materials, when functionalized with $\mathbf{4 - B r}-\mathbf{A r}-\mathbf{N}_{2}{ }^{+}$and is proportional to the degree of functionalization. The solubility of FEG-1 solution was estimated to be $8.13 \mu \mathrm{g} / \mathrm{mL}$ in DMF whereas FEG-2 solution was estimated to be as high as $17.5 \mu \mathrm{g} / \mathrm{mL}$ in DMF, which agrees previous reports in the literature. $[17,18,19]$

To complete the characterizations of the samples, AFM analyses were performed to determine the nature of graphene flaks produced under the cathodic electrochemical method. Figure 2a shows a typical AFM picture of FEG-2, revealing an average lateral dimension of approximately $1 \mu \mathrm{m}$. According to literature reports, the height of bare graphene sheets is around $1 \mathrm{~nm}$ thick [25] as single layer graphene functionalized by substituted aromatic groups display heights around $2 \mathrm{~nm}$ in AFM experiments. [19,26] Statistical AFM thickness analyses of our FEG-2 sample (Figure 1b) show that $\sim 7$ $\%$ of the graphene sheets are $0.7-1.0 \mathrm{~nm}$ thick corresponding to unmodified single layer graphene sheets, $\sim 11 \%$ are $2.0 \mathrm{~nm}$ thick ascribed to monolayer functionalized graphene sheets, $\sim 50 \%$ are 2.7 $3.0 \mathrm{~nm}$ thick assigned to bi-layer functionalized graphene sheets and $\sim 7$ are $3.8 \mathrm{~nm}$ thick ascribed to tri-layer functionalized graphene sheets. These data show that in the FEG-2 sample, nearly $70 \%$ of graphene sheets are composed of mono, bi and tri-layer functionalized graphene sheets.

a)

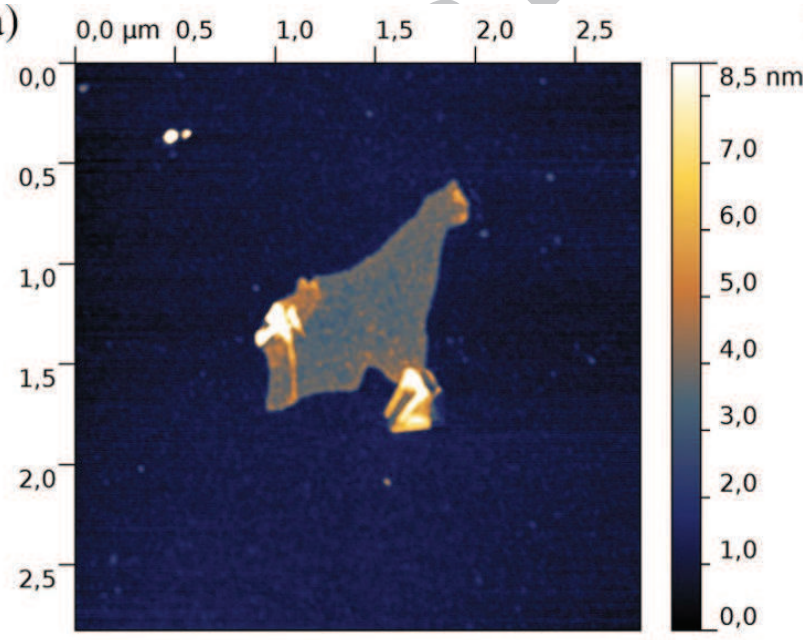

b)

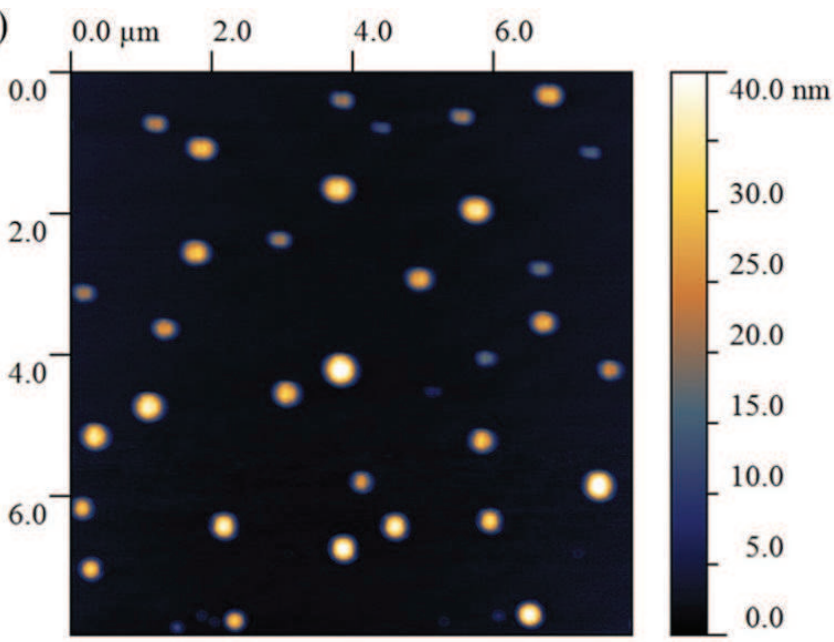

Figure 2. Typical AFM images of a representative a) graphene flak produce by electrochemical expansion of HOPG in DMF and b) carbon particles produce by electrochemical cathodic exfoliation of flexible graphite. 
As HOPG is an expensive carbon source for graphene production, comparative experiments were performed using flexible graphite sheets as carbon source. It appears that during the cathodic electrochemical expansion step, the flexible graphite strips exfoliated instead of expanded. Figure 3 shows the two different carbon sources, HOPG and flexible graphite, after electrochemical cathodic treatment in DMF solution containing $0.1 \mathrm{M}$ tetra- $n$-octylammonium ions. Atomic Force Microscopy (AFM) experiments were performed to determine is the obtained carbonaceous solution was composed of graphene flaks. Unfortunately, as seen on Figure 2b, the obtained carbon particles are as high as 40 $\mathrm{nm}$, which clearly indicated that we are in presence of graphite-like particles and not graphene flaks, highlighting the importance of an adapted choice of the carbon source.

\section{a)}

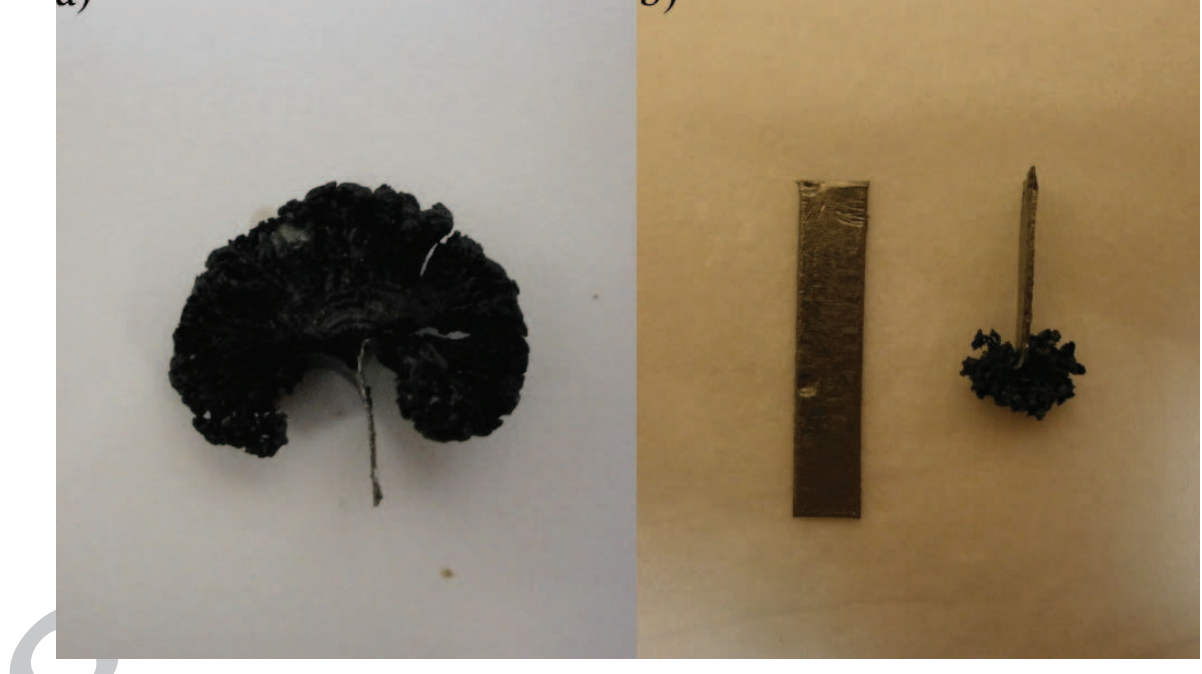

Figure 3. a) Expanded HOPG and b) flexible graphite in DMF solution containing $0.1 \mathrm{M}$ tetra- $n$ octylammonium bromide during 75 min.

\section{Conclusion.}

Cathodic electrochemical treatment of graphite in a DMF solution containing alkykammonium ions is an efficient and fast way for preparing functionalized graphene sheets in minutes, without the need of extended ultrasonic treatment that often alters the structure of the prepared graphene sheets. Introduction of defects in the $\mathrm{sp}^{2}$ carbon lattice, mainly by incorporation of oxygen atom, is also limited 
in these conditions, compared to other classical electrochemical exfoliation methods that are performed in oxidation. The obtained graphene sheets are easily functionalized by the introduction of aryl diazonium ions in the DMF solution, which enhanced their solubility in classic organic solvents. Electro-grafting of aryl diazonium salts is known to be very versatile and thus functionalization of the graphene flaks could be easily extended to other specific functional groups or molecules. For specific applications, one could easily adapt the experimental conditions by tuning the alkykammonium ion used to expand the graphite carbon source and the applied potential. It is noticeable that pyrolytic graphite appears as the most appropriate carbon graphite source to produce graphene sheets by cathodic electrochemical expansion. Tetra-n-octylammonium cations are easily intercalated in HOPG, while the flexible graphite is exfoliated in the same conditions as highlighted by the length diminution of the flexible graphite strips after this treatment.

\section{References}

[1] Novoselov, K.S. Geim, A. K. Morozov, S. V. Jiang, D. Zhang, Y. Dubonos, S. V. Grigorieva, I. V. Firsov, A; A. Science (2006), 306, 666.

[2] Katsnelson, M. Mater. Today (2007), 10, 20-27.

[3] Jong-Hyun Ahn et al. Nano Lett. (2010) 10, 490-493.

[4] Jong-Hyun Ahn et al. Nat. Nanotechnology (2010) 5, 574-578.

[5] Tour, James M. et al. ACS Nano (2012) 6(10), 9110-9117.

[6] Ruoff, Rodney S. et al. Nature (2007) 448, 457-460.

[7] Hummers, W. S.; Offeman, R. E. J. Am. Chem. Soc. (1958) 80, 1339-1339.

[8] Coleman, Jonathan S. et al. Nature Nanotechnology (2008) 3, 563-568.

[9] C.-J. Shih, S. Lin, M. S. Strano and D. Blankschtein, J. Am. Chem. Soc. (2010) 132, 14638-14648.

[10] Coleman, Jonathan S. et al. J. Am. Chem. Soc. (2009) 131, 3611-3620. 
[11] Li, Lain-Jong et al. ACS Nano (2011) 5(3), 2332-2339.

[12] a) Mullen, K. et al. ACS Nano (2013) 7(4), 3598-3606. b) Mullen, K. et al. J. Am. Chem. Soc. (2014) 136, 6083-6091.

[13] Y. Yang, F. Lu, Z. Zhou, W. Song, Q. Chen and X. Ji, Electrochim. Acta (2013) 113, 9-16.

[14] Loh, Kian P. et al. J. Am. Chem. Soc. (2011) 133(23), 8888-8891.

[15] Dai, Hongjie et al. Nature Nanotech. (2008) 3, 538-542.

[16] M. Zhou, J. Tang, Q. Cheng, G. Xu, P. Cui and L.-C. Qin, Chem. Phys. Lett. (2013) 572, 61-65.

[17] Swager, Timothy M. et al. J. Am. Chem. Soc. (2012) 134(43), 17896-17899.

[18] Tour, James M. et al. Nano Res. (2010) 3, 117-125.

[19] Hirsch Andreas et al. Nat. Chem. (2011) 3, 279-286.

[20] D. Aurbach, Non-aqueous Electrochemistry, Marcel Dekker, 1999, ISBN : 0-8247-7334-9.

[21] Hernandez, Y. et al. Nature Nanotech. (2008) 3, 563-568.

[22] Simonet, J. and Lund, H. J. Electroanal. Chem. (1977) 75, 719-730.

[23] A. J. Cooper, M. Velický, I. A. Kinloch and R. A. W. Dryfe, J. Electroanal. Chem. (2014) 730, $34-40$.

[24] K. Kinoshita, Carbon, Electrochemical and Physicochemical Properties, John Wiley \& Sons, Inc., 1988.

[25] a) Ruoff, R. S. et al. J. Mater. Chem. (2006) 16, 155-158. b) Kern, K. et al. Nano Lett. (2007) 7, 3499-3503. c) Chen, Y. et al. ACS Nano (2008) 2, 463-470.

[26] Tour, James M. et al. J. Am. Chem. Soc. (2008) 130, 16201-16206. 
Highlights:

- Modified-graphene sheets are prepared by cathodic electrochemical treatment.

- Modification of graphene flaks is done by electroreduction of aryl diazonium ions.

- Pyrolytic graphite appears as the most appropriate carbon graphite source. 